\title{
Diagnostic difficulties in periodic Cushing's syndrome
}

\author{
$\mathrm{AB}$ Walker, GP Leese, JP Vora
}

\begin{abstract}
Summary
A 22-year-old black woman presented with symptoms suggestive of Cushing's syndrome three years after chemotherapy for a presumed teratoma with cervical lymphadenopathy. Initially, the absence of clinical signs and the demonstration of two normal $24 \mathrm{~h}$ urinary free cortisols appeared to exclude the diagnosis, but an ectopic adrenocorticotropin-producing thymic carcinoid was subsequently removed surgically. Cushing's syndrome due to ectopic adrenocorticotropin production can be difficult to diagnose, particularly if there is periodic hormonogenesis.
\end{abstract}

Keywords: adrenocorticotropin, thymic carcinoid, Cushing's syndrome.

Cushing's syndrome due to ectopic adrenocorticotropin (ACTH) production was first described in 1928 by Brown ${ }^{1}$ and is recognised to occur in association with small cell carcinoma of the bronchus and carcinoid tumours of the bronchus, pancreas and thymus. Whereas cases with florid signs of Cushing's syndrome may pose little difficulty, the diagnosis may be less clear. ${ }^{2}$ In particular when there is associated periodic hormonogenesis or true cyclical Cushing's, investigations such as urinary free cortisol estimation may be intermittently normal, with consequent false-negative exclusion of the diagnosis. Other standard investigations such as an overnight dexamethasone suppression test would also probably be normal in such instances, emphasising the need to follow up and re-investigate in cases of doubt.

\section{Case report}

A 22-year-old black woman presented initially with a supraclavicular lymph node; histology was suggestive of a teratoma. Thoracic computed tomography (CT) was reported as showing an enlarged thymic remnant only and she received chemotherapy (four cycles of bleomycin, etoposide and cisplatin) and local radiotherapy. Subsequent therapy consisted of prednisolone $10-30 \mathrm{mg}$ daily for two years, after which it was reduced to $5 \mathrm{mg}$ daily.

Three years after her initial presentation, she complained of acne, weight gain, bloating and frequent emotional upset. A random ACTH was $36.8 \mathrm{pmol} / 1$ (reference range $21-11.3$ ) and abdominal CT demonstrated bilateral adrenal enlargement. Endocrine assessment was performed after steroids had been stopped for two weeks. On examination, she was slightly overweight but with no truncal obesity; blood pressure was $140 / 80 \mathrm{mmHg}$; skin thickness was normal; there was no hair thinning, no chemosis and no proximal myopathy; however, there were some striae over both thighs. At that stage, two $24-\mathrm{h}$ urine collections revealed normal urinary free cortisols of 87 and $115 \mathrm{nmol} / 24 \mathrm{~h}$ (reference range: $33-290$ ) and normal cortisol:creatinine ratios of 11 and 16 $\mu \mathrm{mol} / \mathrm{mol}$ (reference range $<25$ ). Urine volumes of 1.4 and 1.61 , respectively, suggested that the collections had been complete. It was felt that a diagnosis of Cushing's syndrome had been excluded and she was discharged.

She was admitted four months later in a psychotic state. She had taken no steroids for four and a half months but her mother had noticed increasing puffiness of her face. On examination, her appearance had changed considerably from four months earlier; she had a Cushingoid appearance with increased weight and plethoric, bloated facies. Blood pressure was $180 / 110 \mathrm{mmHg}$ and there were marked striae over both thighs.

Biochemistry demonstrated a hypokalaemic alkalosis: $\mathrm{K}^{+} 1.9 \mathrm{mmol} / \mathrm{l}$; $\mathrm{pH} 7.55$; standard $\mathrm{HCO}_{3}^{-}$42.0. Pituitary $\mathrm{CT}$ was normal and abdominal CT again demonstrated diffuse bilateral adrenal enlargement. Endocrine investigations at that stage demonstrated a markedly elevated cortisol, both at 09.00 $\mathrm{h}>2330 \mathrm{nmol} / \mathrm{l}$ (reference range: $140-500$ ) and at midnight: $>1450 \mathrm{nmol} / \mathrm{l}$ (reference range: $50-300$ ). Plasma ACTH was also very high, both at $09.00 \mathrm{~h}: 55.2 \mathrm{pmol} / 1(2-11.3)$ and midnight: $28.2 \mathrm{pmol} / \mathrm{l}(2-11.3)$. Urinary free cortisols were now grossly elevated at $10083 \mathrm{nmol} / 24 \mathrm{~h}$.

She was treated with metyrapone and when biochemically euadrenal underwent bilateral adrenalectomy, with considerable clinical improvement. Histology demonstrated bilateral adrenal hyperplasia, the adrenals weighing 6 and $9 \mathrm{~g}$. She was started on hydrocortisone and fludrocortisone replacement therapy. Postadrenalectomy, $09.00 \mathrm{~h}$ plasma ACTH was $43.8 \mathrm{pmol} / \mathrm{l}$, with markedly elevated ACTH precursors at $470 \mathrm{pmol} / 1$ (reference range $<80)$. A CT scan of the thorax demonstrated a lobulated mass in the anterior mediastinum extending from the aortic arch for approximately $5 \mathrm{~cm}$ and very close to the anterior surface of the superior vena cava, with subcarinal, right paratracheal and left axillary 
lymphadenopathy. The appearances were suggestive of a thymic tumour with lymphadenopathy, complicated by radiation fibrosis.

She subsequently underwent removal of the anterior mediastinal mass, with division and removal of the left innominate vein and a gortex graft to the right atrium. Histology was of a predominantly epithelial thymoma with frequent areas of haemorrhage and necrosis, but no features of a teratoma. In view of the diagnosis of ectopic ACTH syndrome, on later review of the histology, features were more in keeping with a diagnosis of a thymic carcinoid; the tumour staining positively for ACTH and neuroendocrine markers.

Further CT scans of the thorax six and 12 months postoperatively, showed no clear residual tumour and changes mostly compatible with postoperative scarring and previous radiotherapy. There was initial biochemical improvement following removal of the thymic tumour, ACTH falling to $19 \mathrm{pmol} / 1$. However, there was a steady rise in ACTH over the subsequent 18 months, suggestive of thymic tumour recurrence, or possibly due to loss of negative feedback inhibition by the previously markedly elevated cortisol levels (table).

\section{Discussion}

The diagnosis of Cushing's syndrome due to ectopic ACTH production from a thymic carcinoid in this patient proved difficult and was delayed by the initial absence of clinical signs and normal estimation of urinary free cortisols on two occasions. True cyclical Cushing's and the probably more common periodic hormonogenesis are recognised to occur in both pituitary-dependent Cushing's disease and ectopic ACTH syndrome, ${ }^{5,6}$ but not in adrenal causes of Cushing's syndrome and may be more frequent than previously thought. ${ }^{7}$ Periodic hormonogenesis may be due to areas of necrosis in the ACTH-secreting tumour, ${ }^{8}$ which could certainly account for intermittent ACTH secretion and thus variable degrees of adrenal stimulation. Others have postulated the existence of a negative-feedback loop wherein very high plasma cortisol levels prior to adrenalectomy tend to inhibit ACTH secretion to some extent. ${ }^{9}$ Certainly in our patient ACTH secretion increased markedly in the 18 months post adrenalectomy, in spite of a reduction in overall thymic tumour bulk (table).

Many endocrinologists would be reassured by two normal $24-h$ urinary free cortisols. In

Table Plasma ACTH levels (reference range $2-11.3 \mathrm{pmol} / \mathrm{l}$ )

\begin{tabular}{lc}
\hline Date & $A C T H(\mathrm{pmol} / \mathrm{l})$ \\
\hline $2 / 92$ & 37 \\
$8 / 92$ & 55 \\
(Adrenalectomy and thymectomy) \\
$10 / 92$ & 19 \\
$3 / 93$ & 75 \\
$10 / 93$ & 156 \\
$4 / 94$ & $>290$ \\
\hline
\end{tabular}

retrospect, the presence of a considerably elevated ACTH and bulky adrenals on CT scan may have been a clue either to repeat the 24-h urinary free cortisols within a short follow-up period, or perform an overnight dexamethasone test. However, these may have also been normal if there were periodic hormonogenesis.

Plasma ACTH levels may be markedly elevated in patients with ectopic ACTH syndrome, however, levels may overlap with those seen in patients with pituitary-dependent Cushing's disease. ACTH precursors appear to be significantly elevated in patients with the ectopic ACTH syndrome. ${ }^{10}$ White et al demonstrated a ratio of ACTH precursors to ACTH of 58:1 in patients with the ectopic ACTH syndrome, compared with a ratio of $5: 1$ in patients with pituitary-dependent Cushing's disease, suggesting that this is a useful marker of the ectopic ACTH syndrome. ${ }^{10}$ In our patient, following adrenalectomy and before thymectomy, the ratio of precursors to ACTH ranged from 11 to 41 .

There has been debate as to whether or not the ectopic ACTH syndrome occurs in association with epithelial thymomas; certainly there can be difficulties in distinguishing the two on the basis of light microscopy alone. All of the original descriptions of ectopic ACTH syndrome in association with thymomas are from before the early $1980 \mathrm{s.}^{3}$ Wick et $a l^{4}$ concluded that ectopic ACTH syndrome did not occur with thymomas: when a diagnosis of malignant thymoma had been made, in all cases where they were subsequently able to review the histology, they demonstrated that the correct diagnosis was in fact thymic carcinoid.

Periodic hormonogenesis can make the diagnosis of Cushing's syndrome, particularly due to ectopic ACTH production, difficult. Elevated plasma ACTH levels or adrenal enlargement should alert the clinician to the diagnosis even in the presence of normal urinary free cortisols; such patients need further follow-up and investigation; CT imaging of the thorax and pituitary may be useful. The ratio of ACTH precursors to ACTH may be useful in distinguishing ectopic ACTH production from pituitary-dependent Cushing's disease.

\section{Learning points}

- a normal 24-h urinary free cortisols does not exclude Cushing's syndrome in cases of periodic hormonogenesis; follow-up investigations may be required

- Cushing's syndrome can occur due to ectopic production of ACTH by intrathoracic carcinoid tumours, thoracic CT is therefore mandatory

- markedly elevated ACTH precursors are useful in distinguishing ectopic ACTH production from pituitary-dependent Cushing's disease

- it is difficult to distinguish malignant thymoma from thymic carcinoid on the basis of light microscopy alone; however, the former is not associated with the ectopic ACTH syndrome 
1 Brown WH. A case of pluriglandular syndrome (diabetes of bearded woman). Lancet 1928; ii: 1022-3.

2 Gartner LA, Voorhess ML. Adrenocorticotropic hormoneproducing thymic carcinoid in a teenager. Cancer 1993; 71: $106-11$.

3 Rosai J, Higa E. Mediastinal endocrine neoplasm, of probable thymic origin, related to carcinoid tumour. Clinicopathologic study of 8 cases. Cancer 1972; 29: 1061-74.

4 Wick MR, Scott RE, Li C-Y, Carney JA. Carcinoid tumour of the thymus. A clinicopathologic report of seven cases with of the thymus. A clinicopathologic report of seven cases with

5 Bailey RE. Periodic hormonogenesis. A new phenomenon. Periodicity in function of a hormone-producing tumour in man. $\mathcal{F}$ Clin Endocrinol Metab 1971; 32: 317-27.

6 Liberman B, Wajchenberg BL, Tambascia MC, Mesquita $\mathrm{CH}$. Periodic remission in Cushing's disease with paradoxical periodic hormonogenesis. $\mathcal{F}$ Clin Endocrinol Metab 1976; 43: $913-8$
7 Atkinson AB, McCance DR, Kennedy L, Sheridan B. Cyclical Cushing's syndrome first diagnosed after pituitary surgery: a trap for the unwary. Clin Endocrinol 1992; 36: $297-300$.

8 Thorner MO, Martin WH, Ragan GE, et al. A case of ectopic ACTH syndrome: diagnostic difficulties caused by intermittent hormone secretion. Acta Endocrinol (Copenh) 1982; 99: 364-70.

9 Estopinán V, Varela C, Riobo P, Dominguez JR, Sancho J. Ectopic Cushing's syndrome with periodic hormonogenesis - a case suggesting a pathogenetic mechanism. Postgrad Med - a case suggesting a pa

10 White A, Stewart PM, Gibson S, Crosby SR, Penn R, London DR. ACTH precursors characterise the ectopic ACTH syndrome (abstract). $\mathcal{f}$ Endocrinol 1992; suppl 132: no 155 .

\title{
Male breast tuberculosis
}

\author{
Chandra Jaideep, Mohan Kumar, Ajay K Khanna
}

\section{Summary}

Tuberculosis of the breast is rare and tuberculosis of the male breast is not a recognised entity. We describe a man with tuberculosis of the breast which was clinically thought to be a malignancy.

Keywords: breast, tuberculosis

The differential diagnosis of a swelling of the adult male breast is either gynaecomastia or carcinoma. We present a case of adult male tuberculous mastitis who presented to us with a lump in the breast, clinically suspicious of malignancy. Attention to the tuberculous nature of the lump was drawn by fine needle aspiration cytology, later confirmed by histopathology. To our knowledge this is the first case report of 'male' breast tuberculosis.

\section{Case report}

A 43-year-old man presented to us with a mildly painful lump in the right breast of three months duration. On examination it was mobile, hard, mildly tender and retro-areolar in position, $4 \times 4 \mathrm{~cm}$ in size with nipple retraction. Ipsilateral lymph nodes were enlarged, discrete, non-tender, mobile and 1$2 \mathrm{~cm}$ in size. No history suggestive of tuberculosis was obtained. The clinical picture was suggestive of malignancy, but fine needle aspiration cytology revealed a epithelioid cell granuloma with lymphohistiocytic aggregates (figure 1). The routine haemogram was within normal limits. The erythrocyte sedimentation rate was $42 \mathrm{~mm} / \mathrm{h}$ by Wintrobes' method. Plain X-ray of the chest did not reveal stigmata of past or present pulmonary tuberculosis.
Excision of the lump by a peri-areolar incision was done under local anesthesia and subsequent histopathologic examination revealed a tubercular granuloma with Langhan's giant cell reaction in the breast parenchyma confirming tuberculosis of the breast (figure 2).

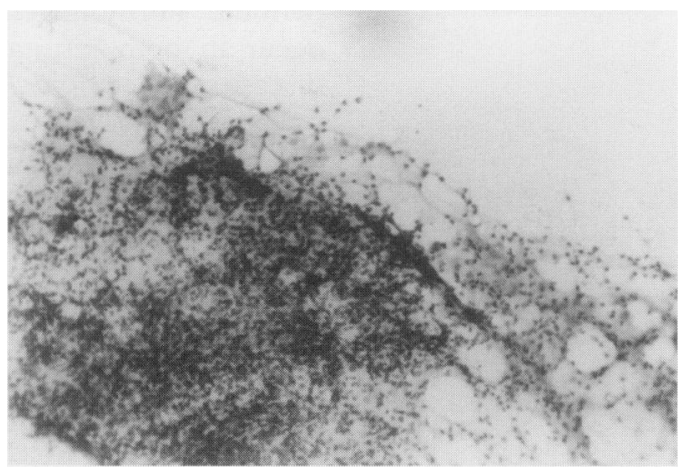

Figure 1 Fine-needle aspiration cytology revealing epithelioid cell granuloma and lymphocytic aggregates (Pap stain $\times 125$ )

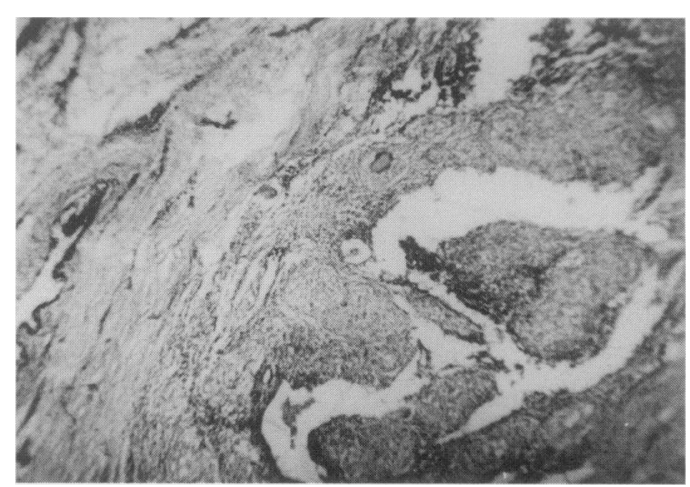

Figure 2 Photomicrograph showing epithelioid granuloma and mammary ducts $(H \& E \times 50)$

\section{Khanna, 33/3, Kabir \\ Nagar, Durgakund, \\ Accepted 21 August 1996 \\ Institute of Medical \\ Hindu University, \\ India \\ Pathology \\ M Kumar}

